\title{
EFEITO DE PARÂMETROS ESTRUTURAIS DO SUBSTRATO SOBRE PREDIÇÕES DE TEMPERATURA E UMIDADE EM BIORREATORES DE FERMENTAÇÃO SÓLIDA
}

\author{
F. P. CASCIATORI ${ }^{1 *}$, A. BÜCK ${ }^{2}$, E. TSOTSAS ${ }^{2}$, J. C. THOMÉO ${ }^{1}$ \\ ${ }^{1}$ Universidade Estadual Paulista, Departamento de Engenharia e Tecnologia de Alimentos \\ ${ }^{2}$ Otto-von-Guericke University Magdeburg, Chair of Thermal Process Engineering \\ e-mail: fernandacasciatori@yahoo.com.br
}

\begin{abstract}
RESUMO
A fermentação em estado sólido (FES) é um processo biotecnológico que consiste no cultivo de microrganismos em matriz sólida porosa úmida. Quando do cultivo em biorreatores de leito empacotado, o sobreaquecimento do leito (devido à geração de calor metabólico) e a secagem do substrato sólido (devido à percolação de ar) são problemas comuns, que limitam a aplicação industrial da FES. Devido às dificuldades de medidas experimentais, modelos matemáticos são utilizados para predizer perfis térmicos e de umidade ao longo do processo. Nestes modelos, as propriedades estruturais da matriz sólida são importantes parâmetros nos balanços de energia e de umidade nos biorreatores de leito empacotado. Neste contexto, o objetivo do presente trabalho foi avaliar o efeito de parâmetros estruturais do substrato sobre as predições, por meio de um modelo heterogêneo bidimensional recentemente proposto, de temperaturas e umidades ao longo de um processo de FES empregando-se o fungo Myceliophthora thermophila I-1D3b, que é bom produtor de enzimas celulolíticas, as quais são essenciais à cadeia de produção do etanol de segunda geração a partir de biomassa lignocelulósica. $\mathrm{O}$ aumento de temperatura foi predito mais intenso em leitos com baixa porosidade, ao passo que a secagem do substrato foi predita mais intensa em leitos com alta porosidade. Portanto, a porosidade exerce efeitos opostos sobre o aquecimento e a secagem do leito, denotando que a operação de biorreatores de FES em leitos empacotados e a escolha do substrato ideal não são tarefas triviais.
\end{abstract}

\section{INTRODUÇÃO}

Desde que o desenvolvimento de uma matriz energética renovável passou a ser visto como estratégico para o Brasil, as enzimas celulolíticas ganharam atenção dos cientistas, tendo em vista aplicação dessas enzimas na hidrólise de materiais lignocelulósicos. É conveniente que a hidrólise seja feita por via enzimática, com vantagens de baixo gasto energético, alta especificidade pelo substrato e não geração de compostos indesejáveis. Todavia, a hidrólise enzimática tem como barreira o custo das enzimas. Uma alternativa interessante para a produção de celulases é a fermentação em estado sólido (FES), processo que permite obter tais enzimas a partir de rejeitos sólidos agroindustriais, o que pode ser econômica e ambientalmente interessante. Como os biorreatores de leito empacotado são de baixo custo e fáceis construção e operação, estes podem ser interessantes para a produção de enzimas por FES. No entanto, esses biorreatores estão sujeitos a aquecimento, devido à geração de calor metabólico, e secagem, devido à percolação de ar.

Os inconvenientes em realizar medidas experimentais em sistemas de FES dificultam 
a ampliação de escala dos reatores, resultando em poucas aplicações industriais destes bioprocessos. Neste contexto, a simulação mostra-se como importante ferramenta, já que, com base em modelos que representem os mecanismos de transferência de calor e de massa nos sistemas de FES, permite prever como o processo é afetado por variáveis operacionais e como as variáveis ambientais afetam o crescimento fúngico e a secreção de bioprodutos.

Modelos para transferência de calor na FES em leitos empacotado têm sido propostos na literatura (SAUCEDO-CASTANEDA et al., 1990; SANGSURASAK; MITCHELL, 1995a; 1995b; 1998), mas informações requeridas para simulação, como propriedades físicas da matriz porosa e características do microrganismo, são frequentemente adaptadas de um sistema biológico para outro, sem profunda consideração acerca de sua validade.

Sangsurasak e Mitchell (1998) foram os primeiros a considerarem, num balanço de energia, a evaporação de água da matriz porosa, que contribui significativamente para a remoção de calor global, mas, em contrapartida, resulta na secagem do leito. A principal deficiência desse modelo é que, sendo pseudo-homogêneo, prediz a remoção de calor evaporativo, mas não prediz a secagem propriamente dita, por não se considerarem fases sólida e gasosa independentes. Modelos heterogêneos, nos quais balanços individuais são propostos para as fases sólida e gasosa, são capazes de representar a secagem do leito ao longo do processo em decorrência da percolação de ar.

Von Meien e Mitchell (2002) foram os primeiros a sugerirem um modelo de duas fases para prever gradientes de temperatura e umidade em um biorreator intermitentemente agitado, tendo modelado apenas os intervalos em que o leito permanecia estático. Apesar de complexo, o modelo de Von Meien e Mitchell (2002) é incompleto, já que é unidimensional e não considera os mecanismos de condução e difusão no leito. Além disso, os coeficientes de interface e as isotermas de sorção do meio foram obtidos da literatura de secagem, não correspondendo ao sistema de FES.

Casciatori (2015) apresentou um modelo de duas fases bidimensional para biorreatores de FES em leito empacotado. Similarmente ao trabalho de Von Meien e Mitchell (2002), foram propostos balanços de energia e de água para as fases sólida e gasosa. Os balanços são representados pelas Equações 1 a 4, respectivamente: balanços de umidade na fase gasosa (Equação 1) e na fase sólida (Equação 2) e balanços de energia na fase gasosa (Equação 3) e na fase sólida (Equação 4):

$$
\begin{aligned}
& \rho_{\mathrm{a}} \varepsilon_{0} \frac{\partial \mathrm{Y}}{\partial \mathrm{t}}+\rho_{\mathrm{a}} \mathrm{v}_{0} \frac{\partial \mathrm{Y}}{\partial \mathrm{z}}=\dot{v}^{\prime}(\mathrm{X}) \beta \mathrm{a} \rho_{\mathrm{a}} \varepsilon_{0}\left(\mathrm{Y}^{*}-\mathrm{Y}\right)+\ldots \\
& \rho_{\mathrm{a}} \varepsilon_{0} \mathrm{D}_{\mathrm{g}, \mathrm{z}} \frac{\partial^{2} \mathrm{Y}}{\partial \mathrm{z}^{2}}+\rho_{\mathrm{a}} \varepsilon_{0} \mathrm{D}_{\mathrm{g}, \mathrm{r}} \frac{1}{\mathrm{r}} \frac{\partial}{\partial \mathrm{r}}\left(\mathrm{r} \frac{\partial \mathrm{Y}}{\partial \mathrm{r}}\right) \\
& \frac{\partial(\mathrm{SX})}{\partial \mathrm{t}}=-v^{\prime}(\mathrm{X}) \beta \mathrm{a}_{\mathrm{a}} \varepsilon_{0}\left(\mathrm{Y}^{*}-\mathrm{Y}\right)+\mathrm{R}_{\mathrm{W}}\left[\frac{\partial(\mathrm{Sb})}{\partial \mathrm{t}}\right]+\ldots \\
& \mathrm{D}_{\mathrm{s}} \mathrm{S} \frac{\partial^{2} \mathrm{X}}{\partial \mathrm{z}^{2}}+\mathrm{D}_{\mathrm{s}} \mathrm{S} \frac{1}{\mathrm{r}} \frac{\partial}{\partial \mathrm{r}}\left(\mathrm{r} \frac{\partial \mathrm{X}}{\partial \mathrm{r}}\right) \\
& \rho_{\mathrm{a}} \varepsilon_{0}\left(\mathrm{Cp}_{\mathrm{a}}+\mathrm{YC} \mathrm{p}_{\mathrm{v}}\right) \frac{\partial \mathrm{T}_{\mathrm{g}}}{\partial \mathrm{t}}+\rho_{\mathrm{a}} \mathrm{v}_{0}\left(\mathrm{Cp}_{\mathrm{a}}+\mathrm{YCp_{ \textrm {v } }}\right) \frac{\partial \mathrm{T}_{\mathrm{g}}}{\partial \mathrm{z}}=\ldots \\
& \Delta \mathrm{H}_{\mathrm{vap}}\left(\mathrm{T}_{\mathrm{s}}\right) \mathrm{v}^{\prime}(\mathrm{X}) \beta \mathrm{a} \rho_{\mathrm{a}} \varepsilon_{0}\left(\mathrm{Y}^{*}-\mathrm{Y}\right)-\mathrm{ha}\left(\mathrm{T}_{\mathrm{g}}-\mathrm{T}_{\mathrm{s}}\right)+\ldots \\
& \varepsilon_{0} \lambda_{\mathrm{g}, \mathrm{z}} \frac{\partial^{2} \mathrm{~T}_{\mathrm{g}}}{\partial \mathrm{z}^{2}}+\varepsilon_{0} \Lambda_{\mathrm{g}, \mathrm{r}} \frac{1}{\mathrm{r}} \frac{\partial}{\partial \mathrm{r}}\left(\mathrm{r} \frac{\partial \mathrm{T}_{\mathrm{g}}}{\partial \mathrm{r}}\right) \\
& \left(\mathrm{Cp}_{\mathrm{s}}+\mathrm{XCp_{ \textrm {w } }}\right) \frac{\partial\left(\mathrm{ST} \mathrm{T}_{\mathrm{s}}\right)}{\partial \mathrm{t}}=\mathrm{ha}\left(\mathrm{T}_{\mathrm{g}}-\mathrm{T}_{\mathrm{s}}\right)+\mathrm{R}_{\mathrm{Q}}\left[\frac{\partial(\mathrm{Sb})}{\partial \mathrm{t}}\right] \ldots \\
& +\left(1-\varepsilon_{0}\right) \lambda_{\mathrm{s}}\left[\frac{\partial^{2} \mathrm{~T}_{\mathrm{s}}}{\partial \mathrm{z}^{2}}+\frac{1}{\mathrm{r}} \frac{\partial}{\partial \mathrm{r}}\left(\mathrm{r} \frac{\partial \mathrm{T}_{\mathrm{s}}}{\partial \mathrm{r}}\right)\right] \ldots \\
& -\Delta \mathrm{H}_{\mathrm{vap}} v^{\prime}(\mathrm{X}) \beta \mathrm{a} \rho_{\mathrm{a}} \varepsilon_{0}\left(\mathrm{Y}^{*}-\mathrm{Y}\right)
\end{aligned}
$$

$\mathrm{Na}$ Equação 1, cada um dos termos representa, respectivamente: à esquerda, a variação de umidade do ar e o arraste de umidade por convecção; à direita, o ganho de 
água por secagem e a difusão de umidade nas direções axial e radial. Na Equação 2, tem-se, à esquerda, a variação da umidade da fase sólida; à direita, respectivamente, a perda de água por secagem, a geração metabólica de água e a difusão de umidade por capilaridade nas direções axial e radial. Na Equação 3, cada termo representa, respectivamente: à esquerda, a variação de entalpia do ar úmido e o transporte de calor convectivo pelo ar; à direita, o calor de evaporação removido da fase sólida, a troca de calor por convecção na interface gás-sólido e a condução de calor nas direções axial e radial. Na Equação 4, tem-se, à esquerda, a variação de entalpia da fase sólida e, à direita, respectivamente, troca de calor por convecção na interface, geração de calor metabólico, condução de calor axial e radial e a remoção de calor evaporativo.

Os avanços do modelo proposto por Casciatori (2015) residem em o modelo ser bidimensional e considerar dispersões térmica e de umidade nas duas fases. A condutividade térmica e a difusividade de água na fase gasosa foram calculadas com base na literatura clássica (TSOTSAS, 2010); para a fase sólida, essas propriedades foram obtidas experimentalmente (CASCIATORI et al., 2013a; MENDES; GUTIÉRREZ, 2014). Coeficientes de interface ha e $\beta$ a foram obtidos por correlações de Nusselt e Sherwood. Isotermas de sorção e densidade e porosidade da matriz sólida foram obtidas para o real sistema de FES tomado como estudo de caso para simulação (CASCIATORI et al. 2014; 2015).

Casciatori (2015) empregara o modelo para prever a distribuição de temperatura, umidade e biomassa fúngica em biorreator de leito empacotado encamisado com 7,62 cm de diâmetro interno e comprimento $1 \mathrm{~m}$, a $45^{\circ} \mathrm{C}$ e com percolação de ar a $240 \mathrm{~L} / \mathrm{h}$ com $85 \%$ de umidade relativa. Biorreator similar fora utilizado experimentalmente para produção de enzimas celulolíticas pelo fungo termofílico Myceliophthora thermophila I-1D3b quando cultivado em mistura de bagaço de cana e farelo de trigo (proporção $7: 3 \mathrm{~m} / \mathrm{m}$ ). Os resultados indicaram secagem do meio nas proximidades da entrada do leito quando da percolação por ar não saturado, limitando o crescimento do fungo naquela região. Para reator estreito, a simulação não indicou sobreaquecimento, devido ao efetivo resfriamento pela parede. Os resultados das simulações concordaram razoavelmente bem com dados experimentais, indicando boa adequabilidade do modelo ao sistema de FES.

A densidade de empacotamento (S) e a porosidade $\left(\varepsilon_{0}\right)$ do leito são importantes propriedades estruturais que aparecem como parâmetros nas equações de balanço de energia e de água nas fases sólida e gasosa do modelo de Casciatori (2015). Ao passo que $S$ aumenta e $\varepsilon_{0}$ diminui, a quantidade de substrato por unidade de volume do biorreator aumenta, aumentando a área superficial disponível para crescimento fúngico, o que consequentemente afeta a transferência de calor e de água entre as fases sólida e gasosa. Valores de $S$ e $\varepsilon_{0}$ dependerão principalmente do tipo de substrato, como forma e tamanho das partículas, de seu conteúdo de umidade e da técnica de empacotamento (CASCIATORI et al., 2014).

Mediante o exposto, o objetivo do presente trabalho foi avaliar o efeito de diferentes combinações de $\mathrm{S}$ e $\varepsilon_{0}$ sobre as previsões de temperaturas e umidades no interior do biorreator de FES em leito empacotado empregado por Casciatori (2015) para produção de enzimas celulolíticas, através do modelo heterogêneo proposto pela mesma autora, visando predizer a viabilidade ou não de se alterarem essas propriedades quando do empacotamento do biorreator, tendo em vista otimização da produtividade.

\section{METODOLOGIA}

O modelo heterogêneo proposto por Casciatori (2015) foi aplicado para previsão 
do perfil de temperatura e de umidade do meio ao longo do processo fermentativo conduzido pela mesma autora para produção de celulases, testando-se quatro combinações entre $S$ e $\varepsilon_{0}$. As simulações foram feitas por meio de programas em MatLab (MathWorks Inc., Natick, USA), considerando-se para os parâmetros operacionais e de processo as condições de referência adotadas por Casciatori (2015).

As combinações testadas entre $\mathrm{S}$ e $\varepsilon_{0}$, respectivamente, foram: $294 \mathrm{~kg} / \mathrm{m}^{3}$ e 0,25 ; $196 \mathrm{~kg} / \mathrm{m}^{3}$ e 0,$50 ; 98 \mathrm{~kg} / \mathrm{m}^{3}$ e 0,75 ; e $59 \mathrm{~kg} / \mathrm{m}^{3}$ e 0,85 . Os valores experimentais de $S$ e $\varepsilon_{0}$ do meio composto por bagaço de cana e farelo de trigo na proporção mássica $7: 3(\mathrm{~m} / \mathrm{m})$ são iguais a, respectivamente, $98 \mathrm{~kg} / \mathrm{m}^{3}$ e 0,75 (CASCIATORI et al., 2014).

\section{RESULTADOS E DISCUSSÃO}

Nas Figuras 1a e 1b, respectivamente, são mostrados os perfis preditos de $T_{s}$ e de $X$ da matriz sólida pelo modelo heterogêneo de Casciatori (2015) para as quatro combinações de $S$ e $\varepsilon_{0}$. Para valores dos demais parâmetros, vide condições de referência em Casciatori (2015).

Para mais altos valores de $\mathrm{S}$ e mais baixos valores de $\varepsilon_{0}$, um aumento de temperatura considerável foi previsto (Figura 1a). A mesma concentração de inóculo inicial, em $\mathrm{kg}$ de biomassa fúngica por $\mathrm{kg}$ de sólido seco inicial, foi assumida para os diferentes $S$ e $\varepsilon_{0}$ correspondentes. Assim, para maiores $S$ e menores $\varepsilon_{0}$, a concentração de inóculo é mais alta em base volumétrica no biorreator ( $\mathrm{kg}$ de biomassa fúngica por $\mathrm{m}^{3}$ ), aumentando a produção de calor por unidade de volume do leito. Entretanto, uma vez que a cinética de crescimento por unidade de massa de substrato não se altera, as propriedades estruturais da matriz sólida afetam mais a magnitude do pico de temperatura do que o tempo que se leva para que a máxima temperatura seja atingida.
Figura 1 - Predições de: (a) $\mathrm{T}_{\mathrm{s}}(\mathrm{z} / \mathrm{L}=0,925$; $\mathrm{r} / \mathrm{R}=0,05) ;(\mathrm{b}) \mathrm{X}(\mathrm{z} / \mathrm{L}=0,075 ; \mathrm{r} / \mathrm{R}=0,05)$ pelo modelo de Casciatori (2015) para diferentes combinações de $S$ e $\varepsilon_{0}$.

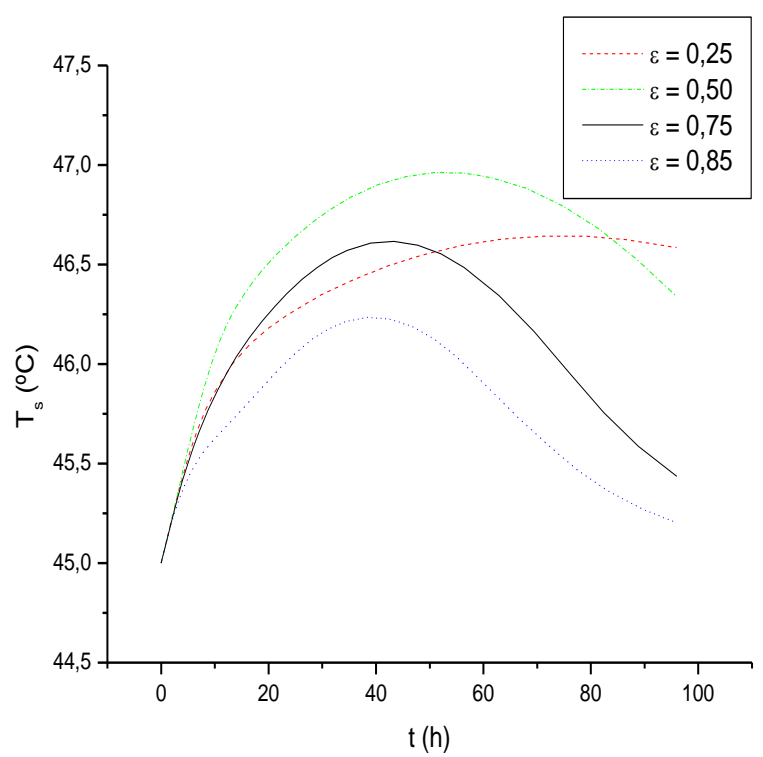

(a)

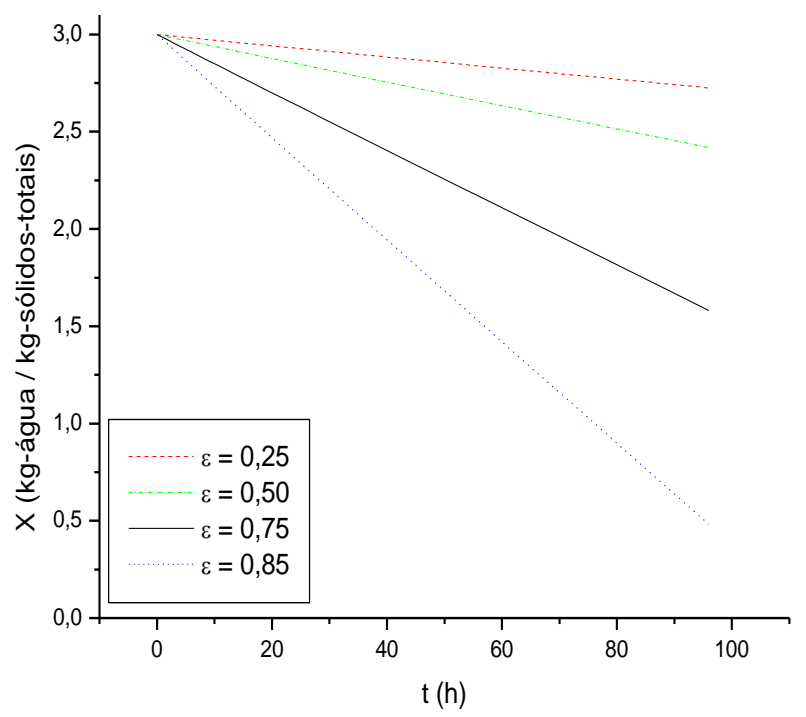

(b)

Tais observações são condizentes com as de Sangsurasak e Mitchell (1998), que já haviam avaliado o efeito de $\mathrm{S}$ sobre os perfis de temperatura previstos por seu modelo 
bidimensional de transferência de calor para o cultivo de Aspergillus niger em farelo de trigo. Aqueles autores já haviam sugerido que avolumar o substrato com algum material inerte poderia ser uma estratégia útil para ajudar a reduzir a severidade do problema de sobreaquecimento no interior de biorreatores de FES em leito empacotado. Por exemplo, para um fungo que metabolize amido, o substrato amiláceo poderia ser misturado com fibras celulósicas, tais como bagaço de cana, reduzindo-se a taxa de produção volumétrica de calor e consequentemente decrescendo o pico de temperatura. No entanto, tal estratégia pode reduzir a produtividade volumétrica do biorreator, sendo conveniente primeiramente testar, em escala de frascos ou de biorreatores de laboratório, o efeito da composição e da densidade de empacotamento do substrato.

No entanto, no presente trabalho, o instante em que o pico de temperatura foi predito a ser atingido também foi alterado para o maior $\mathrm{S}$ e correspondente menor $\varepsilon_{0}$. Diferentemente do modelo de Sangsurasak e Mitchell (1998), o modelo de Casciatori (2015) também leva em conta o balanço de água, que por sua vez também interfere sobre as predições de temperatura no leito, haja vista que um dos mecanismos de remoção de calor é por evaporação.

As predições podem ser consideradas razoáveis tendo em vista que maiores $\mathrm{S}$ e menores $\varepsilon_{0}$ por si sós não são limitantes ao crescimento fúngico, mas interferem na fluidodinâmica do sistema e nos fenômenos de transferência de calor e de água. Por exemplo, para altos $S$ e baixos $\varepsilon_{0}$, a perda de carga ao longo do leito será maior, afetando o escoamento de ar. Além disso, se as partículas são empacotadas de forma muito compacta de modo que possam se deformar e que suas superfícies sejam postas em contato muito próximo, o ar será excluído do espaço intersticial, de modo que as trocas gasosas poderão ser dificultadas. Tais considerações levam a questionar o papel do tamanho e formato das partículas e do tipo de empacotamento na área superficial realmente disponível para o crescimento fúngico na FES (SANGSURASAK; MITCHELL, 1998).

Similarmente, os perfis preditos de $\mathrm{X}$ pelo modelo 2-D também se mostraram fortemente influenciados por $S$ e $\varepsilon_{0}$ (Figura 1b). Baixos valores de $S$ e altos valores de $\varepsilon_{0}$ causaram decréscimo do conteúdo final de umidade do leito, bem como aumento da taxa de secagem. A taxa de remoção de água do substrato aumenta com o decréscimo de $\mathrm{S}$ e acréscimo de $\varepsilon_{0}$ devido ao aumento da área de transferência de água da fase sólida para a fase gasosa por unidade de volume do leito, agravando-se o problema de secagem do substrato devido à percolação de ar não saturado. Além disso, quanto maior o volume de vazios entre as partículas, maior a velocidade intersticial de escoamento do ar, favorecendo-se o aumento da taxa de secagem.

Assim, a escolha da composição do substrato deve levar em conta não somente os aspectos nutricionais do microrganismo, mas também a estrutura do leito, representada por $S$ e $\varepsilon_{0}$, além dos efeitos opostos de $S$ e $\varepsilon_{0}$ sobre $\mathrm{T}_{\mathrm{s}}$ e $\mathrm{X}$, preditos pelo modelo 2-D. Se por um lado leitos mais porosos favorecem o controle térmico, leitos menos porosos são menos sujeitos a secagem. Diante do exposto, o conhecimento das propriedades estruturais das matrizes porosas aplicáveis à FES é fundamental, bem como a simulação dos processos, uma vez que permite avaliar a necessidade de se avolumar o substrato ou aumentar a compactação do leito, de acordo com resultados previstos de picos de temperatura e de quedas de umidade do substrato.

Os resultados de simulação do presente trabalho são condizentes com os experimentais de Casciatori (2015), que cultivou o fungo $M$. thermophila I-1D3b em diferentes substratos em biorreator de leito empacotado com 7,62 cm de diâmetro interno 
e $0,4 \mathrm{~m}$ de comprimento. A autora empregou como substratos uma mistura de fibras de bagaço de cana e farelo de trigo $(7: 3 \mathrm{~m} / \mathrm{m})$, farelo de trigo puro e uma mistura de bagaço de cana em pó e farelo de trigo $(1: 1 \mathrm{~m} / \mathrm{m})$, em termperatura de $45^{\circ} \mathrm{C}$ e com vazão de ar 60 L/h. Casciatori (2015) observou, ao longo do processo, aumento de temperatura expressivo quando do emprego de farelo de trigo puro e da mistura de bagaço de cana em pó e farelo de trigo como substratos, denotando que a baixa porosidade do leito comprometeu a remoção do calor metabólico gerado no interior da matriz sólida nesses casos. Quando do cultivo em substrato contendo fibras de bagaço de cana, meio de maior porosidade, o crescimento do fungo foi bem distribuído pela matriz e a elevação de temperatura foi desprezível, mantendo-se a condição térmica desejável no biorreator.

Diversos outros trabalhos da literatura confirmam os resultados preditos pelo modelo neste trabalho. Casciatori et al. (2014) avaliaram o efeito de diferentes porosidades do leito sobre a produção de celulases (atividade papel de filtro) por Trichoderma reesei QM 9414 cultivado em misturas de bagaço de cana e farelo de trigo nas proporções $1: 1,7: 3$ e 9:1 (m/m) com 70, 75 e $80 \%$ de umidade (em base úmida). A menor atividade foi obtida para a composição bagaço e farelo $1: 1(\mathrm{~m} / \mathrm{m})$ com $80 \%$ de umidade, enquanto a maior atividade ocorreu com composição de bagaço e farelo 9:1 (m/m) com umidade $70 \%$. O procedimento experimental adotado para umidificação do substrato foi adicionar água imediatamente antes de se iniciar o cultivo. Assim, a agua não chegou a se impregnar no material sólido, mas se depositou sobre as partículas na forma de um filme líquido ou preencheu o espaço vazio no leito. No entanto, se sabe que a condição ideal é aquela em que os vazios não estão bloqueados pela água, facilitando as trocas gasosas. Desta forma, explica-se o resultado de o meio com maior teor de fibras de bagaço, mais poroso, e com menor conteúdo de umidade, com espaços vazios não bloqueados pela água, ter favorecido a produção de celulases pelo fungo.

Martin et al. (2004, 2010) testaram várias proporções de bagaço de cana, farelo de trigo e bagaço de laranja para a produção de celulases pelo fungo termofílico Thermomucor indicae-seudaticae N-31 em cultivo sólido. A composição ótima do meio foi bagaço de cana, farelo de trigo e bagaço de laranja 1:2:2 $(\mathrm{m} / \mathrm{m})$, resultando em atividade de pectinase maiores que as obtidas em bagaço de laranja puro e em bagaço de laranja com farelo de trigo 1:1 (m/m). Embora se saiba que o bagaço de laranja é o indutor da produção de pectinases e o farelo de trigo é o iniciador da fermentação, o bagaço de cana favoreceu a produção das enzimas por conferir maior porosidade ao leito.

Barrios-Gonzalez et al. (1993) estudaram o efeito da densidade de empacotamento sobre a produção de penicilina por Penicillium chrysogenum Wis. 54-1255 em FES, tendo bagaço de cana como suporte inerte impregnado com clado de glicose e ácido fenilacético. Os autores concluíram que o uso de particulas maiores de bagaço de cana aumentou a produção de penicilina em $37 \%$, o que foi atribuído ao aumento da porosidade.

Em síntese, para as partículas de tamanho e forma irregulares aplicadas como substratos em processos de FES, não é possível predizer a porosidade com acurácia por equações simples, sendo conveniente realizar medidas experimentais, haja vista o efeito significativo desses parâmetros sobre a previsão de modelos, com comprovação experimental.

\section{CONCLUSÃO}

Tendo em vista os resultados obtidos, reitera-se a importância das propriedades estruturais do substrato na predição de 
temperaturas e umidades pelo modelo heterogêneo bidimensional. Como ambas as predições do modelo são fortemente afetadas pela densidade de empacotamento e pela porosidade do leito, denota-se que sua determinação experimental ou estimativa deve ser conduzida de maneira criteriosa e específica para os substratos empregados na FES, haja vista a confiabilidade das previsões do desempenho dos biorreatores de leito empacotado por simulações numéricas.

\section{NOMENCLATURA}

\begin{tabular}{|c|c|c|}
\hline$b$ & $\begin{array}{l}\text { Fração de biomassa } \\
\text { fúngica na fase sólida }\end{array}$ & $\begin{array}{c}\text { kgbiomassa/ } \\
\text { kg-sólidos }\end{array}$ \\
\hline $\mathrm{Cp}_{\mathrm{a}}$ & Calor específico do ar & $\mathrm{J} / \mathrm{kg}-\operatorname{ar} /{ }^{\circ} \mathrm{C}$ \\
\hline $\mathrm{Cp}_{\mathrm{s}}$ & $\begin{array}{l}\text { Calor específico da fase } \\
\text { sólida }\end{array}$ & $\begin{array}{c}\mathrm{J} / \mathrm{kg}- \\
\text { sólidos } /{ }^{\circ} \mathrm{C}\end{array}$ \\
\hline$C \mathrm{p}_{\mathrm{v}}$ & $\begin{array}{l}\text { Calor específico do vapor } \\
\text { d'água }\end{array}$ & $\begin{array}{c}\mathrm{J} / \mathrm{kg}- \\
\text { vapor } /{ }^{\circ} \mathrm{C}\end{array}$ \\
\hline$C \mathrm{p}_{\mathrm{w}}$ & Calor específico da água & $\begin{array}{l}\mathrm{J} / \mathrm{kg}- \\
\text { água } /{ }^{\circ} \mathrm{C}\end{array}$ \\
\hline $\begin{array}{l}D_{g, z} \\
D_{g, r}\end{array}$ & $\begin{array}{l}\text { Difusividade de água no ar } \\
\text { nas direções axial e radial }\end{array}$ & $\mathrm{m}^{2} / \mathrm{s}$ \\
\hline $\mathrm{D}_{\mathrm{s}}$ & $\begin{array}{l}\text { Difusividade de água na } \\
\text { fase sólida por } \\
\text { capilaridade }\end{array}$ & $\mathrm{m}^{2} / \mathrm{s}$ \\
\hline f & $\begin{array}{l}\text { Variação da umidade de } \\
\text { saturação do ar em função } \\
\text { da temperatura }\end{array}$ & $\begin{array}{l}\text { kg-água/ } \\
\text { kg-ar/ } /{ }^{\circ} \mathrm{C}\end{array}$ \\
\hline ha & $\begin{array}{l}\text { Coeficiente volumétrico } \\
\text { de transferência de calor } \\
\text { na interface gás-sólido }\end{array}$ & $\mathrm{W} / \mathrm{m}^{3} /{ }^{\circ} \mathrm{C}$ \\
\hline $\mathrm{L}$ & $\begin{array}{l}\text { Comprimento total do } \\
\text { reator }\end{array}$ & $\mathrm{m}$ \\
\hline $\mathrm{m} / \mathrm{m}$ & $\begin{array}{l}\text { Proporção massa por } \\
\text { massa }\end{array}$ & $(-)$ \\
\hline & Posição radial & $\mathrm{m}$ \\
\hline $\mathrm{R}$ & Raio total do reator & $\mathrm{m}$ \\
\hline $\mathrm{R}_{\mathrm{Q}}$ & $\begin{array}{l}\text { Coeficiente de geração de } \\
\text { calor por kg de biomassa } \\
\text { produzida }\end{array}$ & $\begin{array}{c}\mathrm{J} / \mathrm{kg}- \\
\text { biomassa }\end{array}$ \\
\hline $\mathrm{R}_{\mathrm{W}}$ & $\begin{array}{l}\text { Coeficiente de geração de } \\
\text { água por kg de biomassa } \\
\text { produzida }\end{array}$ & $\begin{array}{l}\text { kg-água/ } \\
\text { kgbiomassa }\end{array}$ \\
\hline 5 & Densidade do leito & $\mathrm{kg} / \mathrm{m}^{3}$ \\
\hline & & $\mathrm{S}$ \\
\hline$\Gamma$ & Temperatura do leito & ${ }^{\circ} \mathrm{C}$ \\
\hline
\end{tabular}

\begin{tabular}{|c|c|c|}
\hline $\mathrm{T}_{\mathrm{g}}$ & $\begin{array}{l}\text { Temperatura da fase } \\
\text { gasosa }\end{array}$ & ${ }^{\circ} \mathrm{C}$ \\
\hline $\mathrm{T}_{\mathrm{s}}$ & $\begin{array}{l}\text { Temperatura da fase } \\
\text { sólida }\end{array}$ & ${ }^{\circ} \mathrm{C}$ \\
\hline$X$ & Umidade da fase sólida & $\begin{array}{l}\text { kg-água/ } \\
\text { kg-sólidos }\end{array}$ \\
\hline Y & Umidade da fase gasosa & $\begin{array}{l}\text { kg-água/ } \\
\text { kg-ar-seco }\end{array}$ \\
\hline $\mathrm{Y}^{*}$ & $\begin{array}{l}\text { Umidade de saturação da } \\
\text { fase gasosa }\end{array}$ & $\begin{array}{l}\text { kg-água/ } \\
\text { kg-ar-seco }\end{array}$ \\
\hline $\mathrm{z}$ & Posição axial & $\mathrm{m}$ \\
\hline$\beta a$ & $\begin{array}{l}\text { Coeficiente volumétrico } \\
\text { de transferência de massa } \\
\text { na interface gás-sólido }\end{array}$ & $1 / \mathrm{s}$ \\
\hline$\Delta \mathrm{H}_{\text {vap }}$ & $\begin{array}{ll}\text { Calor latente } & \text { de } \\
\text { vaporização da água } & \end{array}$ & $\mathrm{J} / \mathrm{kg}$-água \\
\hline$\varepsilon_{0}$ & Porosidade do leito & $(-)$ \\
\hline$\lambda_{\mathrm{s}}$ & $\begin{array}{l}\text { Condutividade térmica da } \\
\text { fase sólida }\end{array}$ & $\mathrm{W} / \mathrm{m} /{ }^{\circ} \mathrm{C}$ \\
\hline $\begin{array}{l}\Lambda_{\mathrm{g}, \mathrm{r}} \mathrm{e} \\
\lambda_{\mathrm{g}, \mathrm{z}}\end{array}$ & $\begin{array}{l}\text { Condutividade térmica da } \\
\text { fase gasosa radial e axial }\end{array}$ & $\mathrm{W} / \mathrm{m} /{ }^{\circ} \mathrm{C}$ \\
\hline$\rho_{\mathrm{a}}$ & Densidade do ar seco & $\mathrm{kg}-\mathrm{ar} / \mathrm{m}^{3}$ \\
\hline$v_{0}$ & $\begin{array}{l}\text { Velocidade superficial de } \\
\text { escoamento do ar }\end{array}$ & $\mathrm{m} / \mathrm{s}$ \\
\hline$v^{\prime}(\mathrm{X})$ & $\begin{array}{l}\text { Taxa normalizada de } \\
\text { secagem }\end{array}$ & $(-)$ \\
\hline
\end{tabular}

\section{REFERÊNCIAS}

BARRIOS-GONZALEZ， J.; GONZALEZ, H.; MEJIA, A. Effect of particle size, packing density and agitation on penicillin production in solid state fermentation. Biotechnology Advances, v. 11, p. 539-547, 1993.

CASCIATORI, F. P. Produção de celulases fúngicas por fermentação em estado sólido: ampliação de escala de biorreatores de leito fixo. 2015. 177p. Tese (Doutorado em Engenharia e Ciência de Alimentos) Universidade Estadual Paulista, São José do Rio Preto, 2015.

CASCIATORI, F. P.; LAURENTINO, C. L.; LOPES, K. C. M.; SOUZA, A. G.; THOMÉO, J. C. Stagnant Effective Thermal Conductivity of Agro-Industrial Residues for Solid-State Fermentation. International 
Journal of Food Properties, v. 16, p. 15781593, 2013.

CASCIATORI, F. P.; LAURENTINO, C. L.; TABOGA, S. R.; CASCIATORI, P. A.; THOMÉO, J. C. Structural Properties of Beds Packed with Agro-Industrial Solid ByProducts Applicable for Solid-State Fermentation: Experimental Data and Effects on Process Performance. Chemical Engineering Journal, v. 255, p. 214-224, 2014.

CASCIATORI, F. P; LAURENTINO, C. L.; ZANELATO, A. I.; THOMÉO, J. C. Hygroscopic properties of agro-industrial solid by-products applied in solid-state fermentation for cellulase production. Industrial Crops and Products, v. 64, p. 114-123, 2015.

MARTIN, N.; GUEZ, M. A. U.; SETTE, L. D.; DA SILVA, R; GOMES, E. Pectinase production by a brazilian thermophilic fungus Thermomucor indicae-seudaticae $\mathrm{N} 31$ in solid-state and submerged fermentation. Microbiology, v. 79, p. 306-313, 2010.

MARTIN, N.; DE SOUZA, S. R.; DA SILVA, R; GOMES, E. Pectinase production by fungal strains in solid-state fermentation using agro-industrial bioproduct. Brazilian Archives of Biology and Technology, v. 47, p. 813-819, 2004.

MENDES, F. B.; GUTIERREZ, A. M. C. Efeito da umidade nas propriedades físicas e estruturais do bagaço de cana. In: XX Congresso Brasileiro de Engenharia Química, 2014, Florianópolis. Anais do XX Congresso Brasileiro de Engenharia Química, Florianópolis, CD-Rom.

SANGSURASAK, P.; MITCHELL, D. A. The investigation of transient multidimensional heat transfer in solid state fermentation. Chemical Engineering Journal, v. 60, p. 199-204, 1995a.

SANGSURASAK, P.; MITCHELL, D. A. Incorporation of death kinetics into a 2-D dynamic heat transfer model for solid state fermentation. Journal of Chemical Technology and Biotechnology, v. 64, p. 253-260, 1995 b.

SANGSURASAK, P.; MITCHELL, D. A. Validation of a model describing twodimensional heat transfer during solid-state fermentation in packed bed bioreactors. Biotechnology and Bioengineering, v. 60, p. 739-749, 1998.

SAUCEDO-CASTAÑEDA,G.;GUTIÉRREZROJAS, M.; BACQUET, G.; RAIMBAULT, M.; VINIEGRA-GONZÁLEZ,G.Heat transfer simulation in solid substrate fermentation. Biotechnology and Bioengineering, v. 35, p. 802-808, 1990.

TSOTSAS, E. Heat and Mass Transfer in Packed Beds with Fluid Flow. VDI Heat Atlas. Berlin: Springer-Verlag, 2010.

VON MEIEN, O. F.; MITCHELL, D. A. A two-phase model for water and heat transfer within an intermittently mixed solid-state fermentation bioreactor with forced aeration. Biotechnology and Bioengineering, v. 79, p. 416-428, 2002.

\section{AGRADECIMENTOS}

Os autores agradecem, pela concessão de bolsas e auxílio para desenvolvimento desta pesquisa, à Fundação de Amparo a Pesquisa do Estado de São Paulo (FAPESP) (processo $\mathrm{n}^{\circ}$ 2011/07453-5; 2012/13939-0 e 2014/23453-3) e ao convênio FAPESP/ CAPES (proc. 2014/25183-3). 